\title{
Blind Spots in the Science of Safety
}

\author{
Bosk, Charles L.; Pedersen, Kirstine Zinck
}

Document Version

Accepted author manuscript

Published in:

The Lancet

DOI:

10.1016/S0140-6736(19)30441-6

Publication date:

2019

License

CC BY-NC-ND

Citation for published version (APA):

Bosk, C. L., \& Pedersen, K. Z. (2019). Blind Spots in the Science of Safety. The Lancet, 393(10175), 978-979. https://doi.org/10.1016/S0140-6736(19)30441-6

Link to publication in CBS Research Portal

\section{General rights}

Copyright and moral rights for the publications made accessible in the public portal are retained by the authors and/or other copyright owners and it is a condition of accessing publications that users recognise and abide by the legal requirements associated with these rights.

Take down policy

If you believe that this document breaches copyright please contact us (research.lib@cbs.dk) providing details, and we will remove access to the work immediately and investigate your claim. 


\section{Blind Spots in the Science of Safety \\ Charles L. Bosk and Kirstine Zinck Pedersen}

Journal article (Accepted manuscript *)

\section{Please cite this article as:}

Bosk, C. L., \& Pedersen, K. Z. (2019). Blind Spots in the Science of Safety. The Lancet, 393(10175), 978-979. https://doi.org/10.1016/S0140-6736(19)30441-6

DOI: https://doi.org/10.1016/S0140-6736(19)30441-6

* This version of the article has been accepted for publication and undergone full peer review but has not been through the copyediting, typesetting, pagination and proofreading process, which may lead to differences between this version and the publisher's final version AKA Version of Record.

Uploaded to CBS Research Portal: August २०२०

(C) 2019. This manuscript version is made available under the CC-BY-NC-ND 4.0 license

http://creativecommons.org/licenses/by-nc-nd/4.0/ 


\section{Blind Spots in the Science of Safety}

\section{Charles L. Bosk}

Professor of Sociology and Anaesthesiology and Critical Care

Senior Fellow Leonard Davis Institute of Health Economic and Policy

University of Pennsylvania

Philadelphia PA

\section{CLB has received support from Guggenheim Foundation}

\section{Kirstine Zinck Pedersen}

Associate Professor, PhD

Department of Organization

Copenhagen Business School

Kilevej 14A

DK-2000 Frederiksberg

In the last quarter of the twentieth century, a number of observers called attention to rising health care costs associated with the emergence of a new 'medical-industrial complex'. With the coming of the corporation to medicine in the USA and of the new public management in the UK, the EU and the WHO, voices within the medical profession worried that the institutional goal of lowering system costs for health care was in conflict with the personal and professional mission of providing care to patients in hospitals.

A major achievement of the seminal US' Institute of Medicine's (IOM) report, To Err is Human: Building a Safer Health System (2000) was the manner in which it resolved the tension between managers' and providers' sometimes contradictory goals and missions. In To Err is Human, a hidden cost of acute care is identified as 'preventable adverse events' and a set of recommendations is forwarded to reduce them. An international community of health policy-makers in the UK, EU, and WHO embraced the IOM recommendations for improving patient safety. 
These recommendations included: Executive leadership to create a 'culture of safety', the development of 'blame-free incident-reporting systems,' the promotion of robust patterns of teamwork, and work processes that stressed Continuous Quality Improvement. A science of safety built on a systems' engineering platform was imported to health care from high-risk industries to reduce 'preventable adverse events.'

The science of safety has become a swiftly emerging transdisciplinary hybrid domain, the goal of which is to integrate theoretical and practical expertise. Experts are drawn from a wide variety of specialties and subspecialties: neuro- and behavioural psychologists of sleep deprivation and its effects on individual and team performance; human factors engineers with expertise in the computerhuman interface; architectural engineers of informational systems, and organizational psychologists with expertise in team building are all needed. Enhanced system engineering has also led to the development of multiple hybrid sciences to act as complements to safety science: namely translational, implementation and regulatory science.

What evidence there is suggests that applying the principles of a science of safety combined with a systems' engineering approach to complex socio-technical medical work has been a daunting task with mixed results. Some success has been reported for efforts that focus on reducing the occurrence of specific events such as hospital-acquired infections, medication errors, or pressure ulcers. At the same time, mortality rates related to preventable adverse events in American hospitals appear to be climbing by a factor of anywhere of 2.5 to 4 . A pattern of decreased morbidity coupled with increased mortality raises questions about the value of safety science in theory and practice. 
Advocates of targeting preventable patient morbidity and mortality as a problem in the science of safety have mainly relied on two alternatives to explain why programs using the science of safety have not produced a more robust reduction in preventable adverse events. The first explanation faults the lack of appreciation for complexity in medicine on the part of early safety scientists. The first iteration of the science of safety was flawed because the systems that initially served as models for safety were, unlike health care, predictable, simple and stable. The aviation industry was frequently referenced as a model. The reduction over time of deaths on US commercial carriers to zero exemplified the complete success of an industry-wide focus on safety. Safety scientists in healthcare now bemoan the fact that avoiding patient crashes is a task of much greater complexity than is preventing them in planes.

The second explanation blames faulty implementation of safety science principles in medical practice. The findings of the science of safety have not been properly implemented for want of appropriate expertise within healthcare or adequate resources to support organizational change. Delivery systems have responded to these deficiencies in multiple ways. Policy makers and organizational managers have created new roles for systems engineers, hired external consultants to update systems, and provided training opportunities for health professionals to become experts in 'systems thinking'. In addition, computer-based health information technologies and patient-based electronic medical records have created new possibilities for monitoring safety and new possibilities for auditing compliance with formal regulations.

But the problem is not only that the science of safety needs theoretical refinement or that implementation of its findings was flawed. Rather, the deficits of the science of safety are the result of a dynamic tension between what theory recommends and practice demands. Blind spots are 
discovered when safety scientists seek to convert theory into practice. They become visible when theory and practice collide rather than mesh. Inattention to three blind spots begins to explain the failure of the collaborative efforts of safety scientists, systems engineers and front-line workers to reduce adverse outcomes deemed preventable by administrators or regulators.

One blind spot is encoded in the belief that all adverse outcomes that are judged preventable retrospectively would have been prevented prospectively had different choices been made, had available data been interpreted differently, or had more robust safety systems been in place. Safety scientists rely on a set of 'objective' performance measures such as rate of hospital-acquired infections, length of stay, or 30-day readmission rate to document compliance with safety standards. In addition, a number of measures such as 'the number of preventable deaths,' 'formally reported critical incidents', 'near misses,' or 'never events' — have also served as measures of a 'culture of safety' in medicine. All these measures require interpretation. Some possess dubious validity. For instance, 'preventability' is never certain; 'near misses' often go unnoticed; critically-ill patients may have stormy, uncertain illness trajectories; and a high number of self-reported critical incidents can be understood as simply a sign of too many safety breaches or, alternatively, it can be interpreted as a sign of an open and healthy culture of reporting incidents.

As a result of using what are understood to be concrete, tangible indicators of performance, the irreducibly intangible and relational elements in health care are effaced. Comparability of performance measures is assumed in diverse work environments nested within complex organizations that serve demographically disparate patient populations. Anonymized data provides answers to questions at a very high level of abstraction. Clinical conundrums, however, occur with an extraordinary level of granularity and specificity. Workers providing health care close the gap 
between the distant abstraction and the patient in front of them using experiential and contextual knowledge.

Experts in the science of safety may confuse accounting for accountability in part, because they confuse data - a cluster of points—with information — the pattern formed by the points. Such confusion raises important questions concerning the consequences intended and unintended, known and unknown of thinking about preventable adverse outcomes in the terms of the measures used to define them.

A second blind spot is encoded in safety engineering's theoretic disdain for the role of human thinking and acting as features of safe system operation. A fundamental belief in the science of safety is that due to human fallibility, systems must be designed to block known human pathways to error. But both systems and humans fail. Just as systems are designed to constrict certain forms of human error, human operators work tirelessly to make sure that 'systems' operate properly. This human vigilance creates cultures of safety. Experts in the science of safety often redesign work in ways that are intended to overcome the known limitations of the human operator but not those of the system itself.

In the early years of the quest for patient safety, the Joint Commission and the World Health Organization requested that leaders of healthcare systems create 'cultures of safety'. Human vigilance is a pre-requisite for this culture. Vigilance in systems is neither self-executing nor created by standardized work processes. As with any other disposition, vigilance needs to be trained, nurtured, habituated and, once so habituated as to be mindless, relearned. While standardization and routinization simplify complex tasks, they potentially create rote behaviour that in some contexts may erode vigilance. 
A third blind spot of safety science is the inability of an objective, impersonal, evidence-based perspective to capture the normative, situated and experiential dimensions of safe care in the clinic. A scientific stance undermines the fact that in the clinic, medicine and the delivery of care is a thoroughly practical and experience-based exercise. Accomplishing safety and avoiding harm is dependent on experienced teams of skilled practitioners and care workers, who, with the support of hospital leadership, are capable of applying general rules to specific situations, and, in doing so translate an impersonal evidence-base into personal care. Achieving safety through redesigned labour processes neglects the fact that these new ways of working are often in dynamic tension with established and often invisible ways of getting work done.

These already existing but invisible work practices comprise informal responsibility structures, wellfunctioning organizational routines and experience-based normative and discretionary practices. Clinical work and care practices that have often been developed locally through trail-and-error and are not easily standardisable, generalizable and measurable but are none the less the precondition for safe and high-quality care and treatment. The science of safety has no tools to capture this type of work and it thereby risks minimizing the importance of the sentiment that care demands.

There is a science of safety to reduce preventable adverse outcomes. But, health care also has an irreducibly relational, experiential and normative element that remains opaque to safety science. The contribution of a kind and reassuring word; a well-delivered appropriately timed disclosure of a bad diagnosis; an experience-based evaluation of a small but important change in a patient's general condition — all are difficult, if not impossible, to capture in a performance metric. Accomplishing safety and avoiding harm depend on discretion, effective teamwork, and local knowledge on how 
things work in specific clinical settings. Finally, the successful practice of a science of safety presupposes in theory what is most difficult to achieve in practice: a stable functioning team capable of wisely adapting general guidelines to specific cases.

References and suggested readings

Bosk, CL. (2003). Forgive and Remember: Managing Medical Failure, second expanded edition. Chicago: University of Chicago Press

Dixon-Woods, M., McNicol, S. \& Martin, G. (2012). Ten Challenges in improving quality in healthcare: lessons from the Health Foundation's programme evaluations and relevant literature. BMJ Quality \& Safety, 21, 876-884.

Kohn, LT., Corrigan, JM. \& Donaldson, MS. (2000). To err is human: building a safer health system. Washington DC.: National Academy Press.

Makary M, Daniel M. Medical error - the third leading cause of death in the US. $B M J$. 2016;353:2139.

Pedersen, K.Z. (2018). Organizing patient safety: Failsafe fantasies and pragmatic practices, Series: Health, Technology and Society, London: Palgrave MacMillan.

Powers, M. 1997. The Audit Society: Rituals of Verification. Oxford: Oxford University Press

Relman A.J. 1980. The New Medical Industrial Complex. New England Journal of Medicine; 303:963-970

Waring, J., Allen, A., Braithwaite, J. \& Sandall, J. (2016). Healthcare quality and safety: a review of policy, practice and research. Sociology of Health \& Illness, 38(2),198-215. 